\title{
State-Dependent Memory: Neurobiological Advances and Prospects for Translation to Dissociative Amnesia
}

\author{
Jelena Radulovic ${ }^{1 *}$, Royce Lee $^{2}$ and Andrew Ortony ${ }^{3}$ \\ ${ }^{1}$ Department of Psychiatry and Behavioral Sciences, Northwestern University, Chicago, IL, United States, ${ }^{2}$ Department \\ of Psychiatry and Behavioral Neuroscience, The University of Chicago, Chicago, IL, United States, ${ }^{3}$ Department \\ of Psychology, Northwestern University, Evanston, IL, United States
}

OPEN ACCESS

Edited by:

Arash Javanbakht, Wayne State University School of Medicine, United States

Reviewed by: Jennifer Strafford Stevens, Emory University School of Medicine, United States

Pascale Gisquet-Verrier, Université Paris-Sud, France

${ }^{*}$ Correspondence: Jelena Radulovic j-radulovic@northwestern.edu

Received: 03 June 2018 Accepted: 15 October 2018 Published: 31 October 2018

Citation: Radulovic J, Lee $R$ and Ortony A (2018) State-Dependent Memory:

Neurobiological Advances and Prospects for Translation to Dissociative Amnesia. Front. Behav. Neurosci. 12:259. doi: 10.3389/fnbeh.2018.00259
In susceptible individuals, overwhelming traumatic stress often results in severe abnormalities of memory processing, manifested either as the uncontrollable emergence of memories (flashbacks) or as an inability to remember events (dissociative amnesia, DA) that are usually, but not necessarily, related to the stressful experience. These memory abnormalities are often the source of debilitating psychopathologies such as anxiety, depression and social dysfunction. The question of why memory for some traumatic experiences is compromised while other comparably traumatic experiences are remembered perfectly well, both within and across individuals, has puzzled clinicians for decades. In this article, we present clinical, cognitive, and neurobiological perspectives on memory research relevant to DA. In particular, we examine the role of state dependent memory (wherein memories are difficult to recall unless the conditions at encoding and recall are similar), and discuss how advances in the neurobiology of statedependent memory (SDM) gleaned from animal studies might be translated to humans.

Keywords: dissociative amnesia, state-dependent memory, episodic memory, neuronal oscillations, neuronal connectivity, animal models, excitation/inhibition dynamics, stress

\section{INTRODUCTION}

Normal states of consciousness involve an ongoing awareness of oneself and one's environment. Nevertheless, many everyday experiences such as daydreaming, losing track of time, being submerged in a play, a novel, or a movie, are manifestations of temporary dissociation from normal states of consciousness. Getting into, and especially getting out of these states is typically relatively easy, so they are usually considered to be normal. However, in some individuals, particularly those who have been exposed to psychological trauma, dissociation occurs unconsciously and cannot be controlled. In such pathological cases, dissociation is viewed as a "disruption of and/or discontinuity in the subjective integration of one or more aspects of psychological functioning, including-but not limited to-memory, identity, consciousness, perception and motor control" (Spiegel et al., 2011b). Some researchers believe that by compartmentalizing these psychobiological functions, trauma-related threats and distress can be separated from conscious awareness, preventing the experience of pain, discomfort and anxiety, and promoting coping and survival in the face of overwhelming traumatic stressors (Putnam, 1989; Herman, 1992). From this perspective, dissociation can be viewed as adaptive. However, dissociation is maladaptive when it persists and is used to cope with everyday stressors that do not pose a significant threat (Haugaard, 2004; 
Schimmenti and Caretti, 2016; Choi et al., 2017). Under these conditions, dissociation can disrupt the development of self-regulatory processes in stress response systems and can lead to the development of persistent self-dysregulation and dissociative disorders (Curtois and Ford, 2009).

Based on the most prevalent symptomatology, dissociative disorders include dissociative amnesia (DA, inability to access important autobiographical and other memories), dissociative identity disorder (fragmentation of identity and formation of multiple personalities), and depersonalization-derealization disorder (detachment from self or the environment; Spiegel et al., 2011a). DA, which used to be called memory repression, is a manifestation of dissociative disorders that predominantly affects memory systems and enables individuals to detach from the past. The earliest theoretical accounts of such memory failures proposed that overwhelming stress prevents the adequate integration of traumatic and normal conscious experiences (Janet, 1889). Importantly, it was also recognized that although the trauma-related memories were inaccessible their continued existence was manifested through affective and behavioral symptoms (Janet, 1889; Breuer and Freud, 1955). Newer accounts similarly define DA as a process whereby individuals automatically lose access to (dissociate from) memories of an entire traumatic event or details of that event, resulting in significant memory gaps or in no memory at all (Wolf and Nochajski, 2013). DA can also generalize to identity and life history, causing clinically significant distress or impairment in social, occupational, affective and other important areas of functioning. Consistent with the view that dissociation is maladaptive, peritraumatic dissociation is seen as a strong risk factor for the development of PTSD (Briere et al., 2005). However, in some cases DA can also be protective, as evidenced in survivors of childhood sexual abuse suffering from DA who had less depression and anxiety than survivors without it (Coifman et al., 2007).

While improving memory access in the case of generalized DA is an important therapeutic goal, accessing specific traumatic memories can have unpredictable consequences. For some patients, it delays recovery and worsens symptoms. For example, although survivors of childhood sexual abuse suffering from DA tend to suffer less from depression and anxiety, if their memories of the traumatic event surface, these individuals are at increased risk of experiencing higher levels of traumatic symptoms compared to survivors who have never experienced DA (Bonanno et al., 2003). Similarly, the successful recall of traumatic memories can sometimes be highly stressful and can cause symptoms of PTSD or suicidal urges (Fetkewicz et al., 2000). In contrast to these observations, in other individuals, regaining access to trauma-related memories results in positive outcomes because once the memories have been accessed, psychotherapy can help such individuals understand how trauma caused their amnesia, how it disrupted their lives, and how their issues can be resolved so as to help prevent further traumarelated symptoms in the future (Staniloiu and Markowitsch, 2014; Sharma et al., 2015; Cassel and Humphreys, 2016; Markowitsch and Staniloiu, 2016).
Several mechanisms have been proposed to explain restricted access to unwanted traumatic memories. Historically, the idea was that such memories are voluntarily repressed (Breuer and Freud, 1955) - a view that has recently been re-conceptualized as executive control of memory access (Anderson and Green, 2001; Anderson et al., 2004). An alternative view proposes an automatic process wherein such memories are statedependent in that their accessibility is critically dependent on the congruence between the encoding and retrieval conditions (Putnam, 1989; Eich, 1995; Harrison et al., 2017). Although both mechanisms might be playing a role, here we will focus on the relationship between state-dependent memory (SDM) and DA because of recent progress in the understanding of the neurobiology of SDM. The remainder of this article is divided into four main sections. First, we present a case study of an individual, which provides a vivid example of DA ("A Case Study" Section). We then, in Section "SDM as a Gateway to DA-the Human Cognitive Perspective", move on to a discussion of the cognitive foundations of SDM and, especially, of episodic memory, and lay out their relation to DA. Findings from animal and human research emphasizing neurobiological aspects of SDM are reviewed in Section Memory and SDM-a Neurobiological Perspective. In Section Implications of Neurobiological Research for Human DA", we return to the human level and present a brief discussion of the implications of our current understanding of current neurobiological knowledge for DA, after which, in Section "Revisiting Skepticism Concerning DA", we conclude with a discussion of some standard objections to the concept of DA itself.

\section{A CASE STUDY}

The complexity of stress-related disorders can best be illustrated by an example of a clinical case that demonstrates the impact of psychological trauma. The case we have chosen illustrates several common features of DA including high comorbidity with other mental disorders, an inability to recall a life-threatening traumatic memory that was readily recalled by family members, the occurrence of flashbacks after withdrawal from a hypnotic drug (clonazepam) treatment, and full recovery of the memory accompanied by significant clinical improvement following prolonged exposure (PE) psychotherapy.

"Patient $X$ is a 60-year-old male who presented with new onset symptoms consistent with PTSD with dissociative symptoms, delayed expression (309.81 (F43.10)). The index trauma was a house fire 15 years ago, in which he evacuated his granddaughter and sister-in-law while suffering from smoke inhalation. Symptoms included nightmares of the fire, recurrent and involuntary memories of the event, dissociative symptoms including flashbacks, DA in the form of an inability to readily recall details about the fire, avoidance of talking about the fire, profound guilt, severe insomnia and hyperarousal. Of interest, the re-experiencing symptoms did not begin until the present time, a decade and a half after the fire, although hyperarousal symptoms have been chronic. The recent onset of nightmares, reexperiencing and dissociative flashbacks was temporally correlated 
with a reduction and elimination of clonazepam, used to treat a longstanding severe insomnia that was resistant to Cognitive Behavioral Therapy (CBT) for insomnia. His medical history is significant for a history of childhood epilepsy and remote, pre-trauma history of left middle cerebral artery with sparing of the medial temporal lobes but damage to the left parietal lobes. His Montreal Cognitive Assessment (MoCA) score is 29 , with no deficits in declarative or procedural memory, but a mild dysarthria. There is an additional adult history of psychogenic non-epileptic seizures, confirmed by inpatient video EEG recordings of grand mal and partial seizures without correlated EEG activity. He has been on long term anti-epileptic treatment that has remained stable through the period from the fire to the present, including phenobarbital $120 \mathrm{mg}$ qHS and gabapentin $600 \mathrm{mg}$ TID. His psychiatric history is extensive, with a diagnosis of borderline personality disorder since adolescence, with multiple inpatient psychiatric hospitalizations for suicidal ideation and at least three suicide attempts.

Chart review reveals prominent DA regarding the fire. About 1 week after the fire, he was hospitalized at two different hospitals, for nearly 2 months for intractable seizures. It was only during the second hospitalization that the diagnosis of psychogenic non-epileptic seizures was made. Extraordinarily, no mention was made during this 2-month period of the fire by the patient nor the medical chart, despite psychiatric consultations for suicidality. The patient retrospectively reports that from the beginning of the neurological hospitalization to the present day, he has not thought about the fire and has not discussed it with any of his health providers. His subjective experience of that time is of "waking up" in the hospital after a period of sedation in a state of shock, but without memory, or awareness of memory, of the fire. Interestingly, the family corroborates the account of the fire by the patient. His PTSD ChecklistCivilian Version (PCL-C) score was 64, exceeding proposed thresholds for clinically significant PTSD severity. A course of weekly PE psychotherapy was initiated. PE is an evidenced based treatment for PTSD with established efficacy (reviewed in Foa, 2006). During initial imaginal exposure sessions, the level of fear-related arousal was relatively low. The predominant source of distress was shame at not being able to prevent the fire. After six sessions, during imaginal exposure, the levels of distress related to fear increased, to subjectively maximal levels of intensity (100\% of experienced fear intensity). Simultaneously, the PCL-C scores began to decrease to 55. During session seven, he had a short seizure during imaginal exposure, coinciding with peak subjectively experienced fear. The seizure was consistent with previous psychogenic non-epileptic seizures. During session eight, he revealed that in the previous week, practicing imaginal exposure at home resulted in psychogenic non-epileptic seizures about $50 \%$ of time with prolonged periods of dissociative state. In session eight, session imaginal exposure was modified to be conducted with eyes open rather than closed. Additionally, when dissociative symptoms started, verbal reorienting was provided, followed by resumption of imaginal exposure. He was to practice imaginal exposure at home with his lapdog present, who has been trained to lick the patients face at signs of dissociation or seizure. At session nine, the PCL-C score for the previous week decreased further to 41 , with a marked reduction in frequency of nightmares. He was able to complete PE over the course of six more sessions, without a return of seizures during session, and a final PCL-C score of 21.

In summary, this was case of delayed onset of PTSD, with onset of symptoms occurring a decade and a half after the trauma. In the interim, there was documented evidence of DA, which is relatively common in patients suffering from borderline personality disorder (Sar et al., 2014). Family members confirmed the severity of the fire and involvement of the patient. The trigger for the onset of PTSD symptoms was the elimination of a nighttime dose of clonazepam. During a course of $\mathrm{PE}$, there was further reduction of DA, specifically of emotional numbing, which had previously blocked access to the subjective experience of fear of dying due to smoke inhalation and heat exposure. Although access to intense feelings of fear and distress when retrieving of details of the traumatic experience led to transient states of dissociation and even non-epileptic seizures during a session, continuing PE therapy resulted in habituation and reduction of fear and distress. The dissociative states and non-epileptic seizures did not return.

Even though in some cases, as in this one, therapy seems to be successful, in general there is disappointingly little evidencebased research to inform successful approaches to the treatment of DA. This might be due in part to a bitter controversy in the field that arose in the 1990's as to whether DA is a real phenomenon. The controversy, which came to be known as the "Memory Wars" (after the widely publicized book by Crews, 1995), was largely a reaction to psychodynamic approaches to DA (in particular those arising from cases of alleged childhood sexual abuse). Issues of central concern related to "repression" as a specific mechanism of memory inaccessibility (Breuer and Freud, 1955), as well as to the problem of distinguishing false from veridical memories (Loftus and Davis, 2006) and dissociated from non-dissociated memories (McNally, 2007). Fortunately, advances in both human and animal research in the neurobiology of memory are providing new insights in light of which many such questions can be newly addressed.

\section{STATE-DEPENDENT MEMORY AS A GATEWAY TO DA-THE HUMAN COGNITIVE PERSPECTIVE}

In seminal work on the relationship between stress and memory, Brewin et al. (1996) proposed that traumatic experiences give rise to two types of memory representations. One type results from the conscious processing of the trauma and includes accessible memories that can be expressed verbally, while the other results from the unconscious processing of the trauma. Brewin et al. (1996) referred to the result of this latter type of memorial representation as "situationally accessible knowledge," which they argued is automatically retrieved when a person is in a situation that is similar to the one in which the trauma was experienced, a view supported by many others (Eich, 1995; van der Kolk and Fisler, 1995; Whitfield, 1995). 
This account of "situationally accessible knowledge" fits well the definition of the phenomenon of SDM that we discuss in depth below. However, before doing so, we need to set the stage by briefly reviewing some key concepts in human memory.

\section{Memory Systems}

Understanding the basic issues relating to memory ought to be simple: through experience and learning, we acquire information, we encode it, retain it for later use, and when we need it, we retrieve it. But, of course, it isn't that simple, in part because memory is not a unitary concept and such a bare-bones account inevitably neglects its rich complexity. Cognitive psychologists have identified all manner of different kinds of memory-iconic, haptic, echoic, short-term, working, long-term, declarative, non-declarative, procedural, semantic, episodic, implicit, explicit and more. These different kinds of memory, or memory systems, can be thought of as (collections of) different kinds of specific memories, and they are distinguished in terms of the nature of their content, their durability, and the way in which they are acquired and accessed.

The most relevant top-level aspect of the human memory system is long-term memory, which comprises information that is retained for a long time-days, weeks, months, or years, rather than seconds, minutes, or hours. Long-term memory is comprised of non-declarative and declarative memory (Squire, 1992), a distinction which is reminiscent of the classic partitioning of knowledge into knowing how and knowing that (Ryle, 1945). The brain has the capacity to store vast amounts of information that is used to organize behavior and make decisions, and much of this information is part of the non-declarative memory system, which means that it can be accessed and used automatically without the need to voluntarily retrieve it. Non-declarative memory includes procedural memory (or knowledge) such as one's knowledge of how to ride a bicycle, as well as the results of simple classical conditioning, of perceptual learning, and of non associative learning (e.g., habituation).

By contrast, declarative memories are records of specific facts and events that can normally be intentionally recalled. Memories of facts and events can be talked about, they can be articulated in language, they can be reported; hence, "declarative." The declarative memory system consists of episodic memory, with which this article is primarily concerned, and semantic memory. Individual episodic memories are representations of actual experiences that generally incorporate the spatial, sensory, and temporal information associated with those experiences, integrated into a unitary whole. In its original formulation (Tulving, 1972), the episodic memory system was characterized as the totality of a person's encoded personal experiences-an autobiographical record of experienced events and their temporal and spatial contexts. Subsequently, Tulving (1985) modified the idea, tying it more explicitly to the conscious act of remembering a past experience. On the revised view, for something to count as an episodic memory it was not sufficient that the remember merely know (or believe) that something happened to him or her. That kind of factual knowledge, even though it is knowledge about the self, is better thought of as belonging to semantic memory. Rather, Tulving proposed that the construct of episodic memory capture the awareness-the autonoetic consciousness-associated with the actual act of remembering, for it is this that bestows the "special phenomenal flavor to the remembering of past events" (p. 3). On this view, what matters is the remembering of the experience itself rather than the remembering of the fact of the experience (Markowitsch and Staniloiu, 2016).

\section{The (in)fidelity of Episodic Memories}

An important aspect of individual memories is their degree of fidelity. Fidelity has to do with the relation between what was experienced and what was encoded, and between what was encoded and what was retrieved, and thus concerns the integrity of the information encoded or retrieved. It is well-established that memories of meaningful information are rarely exact records of what was seen or heard (Bartlett, 1932; Bransford and Franks, 1971; Anderson and Ortony, 1975). In general, there is very little information that is encoded and retained verbatim. Instead, even at the time of encoding, what is encountered routinely contains omitted as well as elaborated and even intruded information, often the result of unconscious inferences. Thus, what is encoded is not the raw sensory or semantic input, but a representation constructed from that input. Furthermore, just as encoding is a constructive process, so is retrieval. The most celebrated early exponent of this (re)constructive view of memory is Frederick Bartlett who undertook detailed experimental work on memory for drawings and stories. Bartlett's work, and that of many after him, established conclusively that memory for meaningful material normally involves the unconscious elaboration of stored fragments of that material enhanced with general world knowledge, associations and conventional ideas and schemas. In addition, memory for such material is also influenced by subsequent exposure to relevant related information as well as by subsequent successful or unsuccessful attempts to recall it.

The basic principles relating to the constructive nature of memory encoding and retrieval mean that the fidelity of the relation between what was encountered and what is encoded can in no way be guaranteed, nor can the fidelity of the relation between what was encoded and what is or can be retrieved. Thus, even under normal conditions, memory distortions, memory failures, and even false memories are routine psychological phenomena.

\section{Sources of Problems in Accessing Episodic Memories}

The concepts of retrieval and forgetting are, of course, central to any discussion of memory and memory-related disorders. Assuming that forgetting is some sort of failure of retrieval, we can start by asking what retrieval is. One might think that retrieval is simply the process of recovering or locating information stored in memory (VandenBos, 2015). However, this kind of definition is too course-grained to be useful, not 
least because it fails to acknowledge two crucially different processes, namely, intentional vs. unintentional access to stored information-the willful effort to retrieve, also called recall, vs. the incidental, unintended activation of such information, as happens in many cases of recognition and reminding where, material comes to mind unbidden. In our discussion of memory processes in humans, we shall primarily be concerned with the intentional process of retrieval rather than with unintentional processes, and with the nature and consequences of failures of retrieval (i.e., the forgetting) of episodic memories.

Forgetting can occur for one of two general reasons: either the to-be-remembered material itself is compromised, or access to that material is compromised. When the memory itself is compromised, forgetting can occur because its contents have degraded so that only fragments of the original memory remain, or in some cases because the memory is degraded to such an extent that there is nothing coherent to access at all. But, forgetting can also result from a failure of the retrieval mechanism to access an intact memory. In such cases, access to the to-be-recalled representation is for one reason or another, temporarily (or even permanently) blocked, as in the case of patient $\mathrm{X}$, described in the case study, who for 15 years was unable to retrieve his traumatic involvement in a frightening house fire.

Of particular interest in the present context is the kind of retrieval failure that occurs when the conditions at time of retrieval differ from those that pertained at the time of encoding. Although known as SDM, this phenomenon might be thought of as a special case of blocked-access forgetting, because statedependency could be a feature of the access mechanism rather than (or in addition to) a feature of the memory itself. This kind of state-dependent forgetting is particularly important because of its potential relevance to DA. We will therefore now discuss it in greater detail, although referring to it by its more conventional name of SDM.

Formal definitions of SDM hone in on the psychological, biological, or physical states of the rememberer. For example, dictionaries of psychology define SDM as "the tendency for information that was learnt in a particular mental or physical state to be most easily remembered in a similar state" (Colman, 2009 italics added), or as "a condition in which memory for a past event is improved when the person is in the same biological or psychological state as when the memory was initially formed" (VandenBos, 2015, italics added). State dependence is a quite general phenomenon which to some degree is characteristic of many kinds of memories. An interesting anecdotal example of its relevance to cases other than episodic memory, and which is also of some historical interest, is mentioned by Godden and Baddeley (1975). They noted that John Locke, the 17th century British philosopher, wrote of a man who, having learned to dance "to great perfection" in a room in which there was a wooden trunk, could then only perform well what he had learned in that or a similar room, and one in which was situated a similarly placed trunk. This would be an example of state-dependent procedural memory. Statedependency can also arise in simple conditioning. Indeed, the first documented experiment demonstrating SDM (Girden and Culler, 1937) was in the context of a conditioned reflex in dogs, with learning taking place under conditions quite different from normal states of consciousness. These authors showed that a conditioned reflex induced under curare could not be induced at all when the dogs were awake, there being a complete amnestic barrier between the normal and curareinduced states.

Even though it is important to recognize that state dependence is a phenomenon that occurs in the context of other kinds of memory, its occurrence in the context of episodic memories is particularly interesting because episodic memories, reflecting as they do personal experiences, are by definition the kind of memories that can be consciously recalled. In their classic experiment, Godden and Baddeley (1975), referring to their particular case of SDM as contextdependent memory, demonstrated that the recall of word lists that scuba divers had learned under water was superior when the divers were again under water than when they were on dry land. The phenomenon has also been demonstrated when acquisition of the to-be-remembered information occurred under the influence of psychoactive drugs such as alcohol (Weingartner et al., 1976) and marihuana (Hill et al., 1973); and essentially the same phenomenon, but under the label of encoding specificity (Thomson and Tulving, 1970; Tulving and Thomson, 1973) was demonstrated by showing that retrieval of items from episodic memory was optimal when the conditions at the time of retrieval, such as context or available cues, were the same as those at the time of encoding.

It should be noted that the fact that state-dependency is about the optimal conditions for accessing items stored in episodic memory does not mean that absent those conditions, memory access will necessarily fail. In fact, state-dependence is perhaps best be thought of as a variable that can affect the ease of access, ranging from minimal if any influence at one end of the continuum to substantial influence at the other. It might be that cases of SDM in which access to a memory is highly restricted, representing the extreme (high-influence) end of the continuum, are qualitatively different from other cases. These would be the cases of most relevance for DA.

\section{MEMORY AND SDM-A NEUROBIOLOGICAL PERSPECTIVE}

DA, flashbacks and other dissociative phenomena have frequently been observed not only as a result of traumatic stress, but also as a result of the use of dissociative drugs such as PCP, ketamine, or LSD (Brna and Wilson, 1990). However, due to ethical and regulatory issues, such drugs cannot be used in human SDM research. For this reason, most of our current knowledge on the neurobiology of SDM is based on animal studies. Using tools for visualizing and manipulating neurons directly involved in memory processing in animals (Gradinaru et al., 2010; Zhu and Roth, 2014), it is now possible to study the memory circuits that process veridical memories, false memories and SDMs (Garner et al., 2012; Ramirez et al., 2013; Liu et al., 
2014; Jovasevic et al., 2015). We are thus in an unprecedented position to explore the connection between state-dependency and memory access in DA. In fact, animal models allow us to examine extreme cases of SDM, in which memories cannot be retrieved at all under normal conditions ("complete amnestic barrier" Overton, 1991). Below, we discuss the strengths and limitations of animal approaches to episodic-like memories including SDM, highlighting the relevance of emerging findings to our understanding of DA and possibly other memory-related psychopathologies.

\section{Modeling Episodic-Like Memories and SDM in Rodents}

Robust memories of stressful experiences that persist over months or years (Gale et al., 2004) can be readily induced in experimental animals. This is typically done using contextual fear conditioning or passive avoidance learning in which animals-most often, rodents-learn to associate multisensory environmental contexts with aversive foot shocks. As evidence that such learning has occurred, upon re-exposure to the conditioning context, rodents express either freezing behavior (contextual fear conditioning if the animals cannot escape) or avoidance behavior (passive avoidance if they can escape). Such memories resemble human episodic memories in that they require the integration of spatial, multisensory, and temporal information into memory, and this integrated representation has to be accessed for freezing or avoidance behavior to occur (Fanselow, 1990; McGaugh and Roozendaal, 2002). Both in rodents and humans, these memories depend on neuroanatomical mechanisms which differ from those required for conditioning to simple cues (Kim and Fanselow, 1992; Phillips and LeDoux, 1992). The only aspect of human episodic memory that we cannot test in animal models is the subjective re-experiencing of the encoded event, for which reason we use the expression episodic-like memory when discussing animal research. Despite this limitation, the neurobiological basis of the processing of episodic-like memories in animals is known to be surprisingly similar to that in humans (Grillon, 2002; Milad et al., 2006; Rauch et al., 2006) and can thus be successfully used to develop mechanistic hypotheses related to memory processes.

From a neurobiological point of view, memories are most likely encoded and retrieved as sequences of neuronal activity (Eichenbaum, 2000; Hasselmo, 2005; Pastalkova et al., 2008; Carr and Frank, 2012) bound and ordered by neuronal oscillations (Lisman and Jensen, 2013). These patterns of activity vary with neuronal connectivity and other properties, such as strength of synaptic connections and responsiveness to excitatory and inhibitory input, which are determined by the genetic and molecular profile of each neuron. These features change in different brain states, sometimes resulting in state-dependent encoding of memories, as reviewed recently (Radulovic, 2017; Radulovic et al., 2017). Furthermore, it appears that even initially accessible memories can be rendered state-dependent if the brain states are manipulated at the time of retrieval (Sierra et al., 2013; Gisquet-Verrier et al., 2015). Such memories are no longer accessible under the conditions present at encoding, but instead, under the conditions present at retrieval. Thus, there is much evidence that the efficient encoding of memories, but with only limited subsequent access, is possible.

\section{Mechanisms of SDM: the Role of Excitatory/Inhibitory Balance and Stress}

The standard approach to studying SDM is to manipulate the brain states of animals by using various drugs (Netto et al., 1987; Overton, 1991; Colpaert et al., 2001; Rezayof et al., 2003). Such pharmacological approaches have unique advantages such as allowing for rigorous control over the experimental conditions (e.g., dose, injection site, memory phase), and enabling the determination of the role of individual neurotransmitter systems. This means that we can investigate mechanisms that causally contribute to memory processing in different brain states and characterize these states at different scales of neuronal function. Moreover, animal models can be easily designed to test extreme cases of SDM that completely restrict memory access, which could be particularly relevant to DA.

At the level of neurotransmission, the encoding and retrieval of episodic memories depend on the dynamics between neuronal excitation (mediated by glutamate) and inhibition (mediated by GABA; Froemke, 2015). Whereas excitatory neuronal networks are believed to play a key role in memory encoding and retrieval, inhibitory networks have been typically viewed as memory impairing (Rudolph and Möhler, 2006). However, this stance has been challenged by recent evidence indicating that while inhibitory networks do make memories quiescent, those memories nevertheless remain available for activation under particular conditions (Barron et al., 2016, 2017). Consistent with this view, many drugs used to generate SDM in animal models increase the inhibitory tone in the hippocampus (Radulovic et al., 2017). SDM can also be seen at the other extreme, namely under conditions of enhanced excitatory transmission, for example in response to psychostimulants and noradrenaline (Overton, 1991; Berridge and Waterhouse, 2003).

Just as drugs alter the local or global excitatory/inhibitory dynamics, so too do stressful experiences. In some cases, acute stress predominantly triggers release of glutamate (Popoli et al., 2011). In other stress paradigms, there are important individual variations, with some animals responding with low GABA/glutamate release (indicating relatively high excitation vs. inhibition) and others with high GABA/glutamate release (indicating relatively high inhibition vs. excitation) in the prefrontal cortex (Drouet et al., 2015). High inhibition has also been found after chronic stress in both adult (McKlveen et al., 2016) and juvenile (Albrecht et al., 2016) rodents. Notably, the effects of juvenile stress persisted throughout adulthood in a population of hippocampal neurons (dentate granule cells). These findings suggest that under some circumstances, and when stress is particularly severe (Drouet et al., 2015), the excitatory/inhibitory balance can shift towards inhibition or excessive excitation, both of which are more likely to result in SDMs than in easily accessible memories. A shift 
in excitatory/inhibitory balance in the direction of increased excitation could underlie the finding in patient $\mathrm{X}$ described in the case study, whose loss of memory of the fire was alleviated during clonazepam withdrawal, which typically results in overexcitation.

Although the similarities between stress-induced and drug-induced SDM are suggestive, it is important to note that reliable models of stress-induced SDM have yet to be developed and validated. To date, the field has mainly focused on stress-enhanced and extinction-resistant memories (Rau et al., 2005; Tronson et al., 2010) rather than inaccessible memories. Given the advances in our understanding of memory impairing effects of stress (Todorovic et al., 2007; Maras et al., 2014; Moreira et al., 2016), studying inaccessible memories is now feasible. The development of reliable animal models of stress-induced SDM is an important future challenge for the identification of a translational link between fundamental mechanisms identified in animals and psychopathologies in humans.

\section{Brain States That Subserve SDM}

At first glance, the finding that susceptibility to SDM processing increases with the excitation/inhibition balance might suggest that SDM is a quantitative rather than a qualitative phenomenon. However, analyses at the level of network activity and connectivity indicate otherwise. Changes in the hippocampus, which is known to play important roles in processing both conscious and unconscious memories (Henke, 2010; Hannula and Greene, 2012; Shohamy and Turk-Browne, 2013), may be particularly relevant. A notable change within the hippocampus and cortex during SDM encoding under heightened tonic inhibition (using systemic infusion of the drug gaboxadol) is the increase in power of slow, delta oscillations, along with a decrease of theta and gamma oscillations (Meyer et al., 2017). At the same time, at the circuit level, the connectivity between the hippocampus and neocortical areas (retrosplenial, entorhinal and anterior cingulate cortex) is significantly reduced (Jovasevic et al., 2015; Meyer et al., 2017). Consistent with these observations, it was recently found that enhanced cortical delta oscillations causally contribute to the formation of state-dependent fear-inducing context memories during states of reduced excitation (using systemic administration of the NMDA receptor antagonist MK-801; Jiang et al., 2018).

Changes of network activity and connectivity have at least three important implications. First, they are consistent with the suggestion of Jacobs and Nadel (1998) that traumatic levels of stress lead to disconnections between memory processing brain areas. This view has been supported by recent imaging studies in patients with DA (Staniloiu and Markowitsch, 2012; Harrison et al., 2017; Thomas-Antérion, 2017). For example, patients show alterations of functional MRI imaging signals in frontal and temporal lobes (Hennig-Fast et al., 2008) with increased prefrontal and decreased hippocampal metabolic activity during testing for memory recall (Kikuchi et al., 2010). After treatment for DA, the pattern of brain activation normalized in a patient whose memories were recovered, whereas it remained unchanged in the patient whose memories were not recovered. Although these initial findings need to be confirmed in larger cohorts, they suggest a direct relationship between alterations of hippocampal and cortical activity and DA. Thus, as in other cases of memory inhibition (Anderson et al., 2004; Benoit et al., 2015), DA is often accompanied by increased activation in the dorso- and ventral-lateral prefrontal cortex, with associated deactivation in medial temporal structures, such as the hippocampus.

Second, the role of slow oscillations in SDM could explain an apparently paradoxical observation, namely, that access to traumatic memories can be facilitated not only under conditions of elevated stress, as seen in patient X during PE therapy, but also during therapies carried out while in hypnotic or relaxed states (Li et al., 2017). We know that the power of delta waves increases both during elevated arousal associated with severe stress (Kolassa et al., 2007; Ahnaou and Drinkenburg, 2016; Marshall and Cooper, 2017) and during states of sleep and relaxation (Knyazev, 2012). For example, a study of memories acquired under severe stress (near death experiences) found that the power of delta oscillations was positively correlated with memory details recalled during hypnosis, particularly with regard to the resolution, reliving, and spatiotemporal organization aspects of those memories (Palmieri et al., 2014).

Third, the findings pertaining to the relationship between delta oscillations and SDM could further our understanding of the relationship between brain oscillations and memory processes more generally. Both empirical data and computational modeling suggest that at the level of the hippocampus, memories are encoded as sequences (patterns) of neuronal activity that are combined into "chunks" of memories at the level of the cortex (Levy and Wu, 1996; Kesner and Rolls, 2015). Importantly, the size of these sequences depends on the balance between excitatory and inhibitory synaptic connections (Levy and $\mathrm{Wu}, 1996)$. Typically, theta oscillations are highly effective in binding components of episodic memories, however, this does not seem to be the case in human DA or extreme cases of SDM in animals. It is possible that traumatic memories and SDMs are particularly fragmented (van der Kolk and Fisler, 1995; Nadel and Jacobs, 1998) in which case slower delta oscillations might bind them more effectively than theta oscillations. However, this speculation would need to be tested experimentally.

All in all, neuroscience research in rodents demonstrates that depending on the conditions, stress-related experiences can be encoded either as robust memories or as impaired memories. According to our model, tress-related SDMs, as an example of the latter, would be particularly favored when the excitation/inhibition balance is shifted towards extremes, resulting in qualitatively different brain states in terms of brain oscillations and overall connectivity between hippocampal and cortical circuits, as illustrated in our recent work (Radulovic et al., 2017). We suggest that such states are likely to lead to the encoding of memories in more fragmented sequences that cannot be bound with high-frequency oscillations and therefore cannot be easily integrated in the hippocampal-cortical episodic memory circuits. 


\section{IMPLICATIONS OF NEUROBIOLOGICAL RESEARCH FOR HUMAN DA}

Although the phenomenon of SDM has been recognized for a long time, our understanding of its underlying mechanisms is only in its early stages. Nevertheless, with advances in human neuroscience research, several findings from animal research can already be tested, and possibly translated to humans. The increasing sophistication of imaging techniques has paved the way for delineating the processing of real, imagined, and highstress-induced memories (Palmieri et al., 2014). For example, by using dynamic causal models derived from data from EEG and fMRI studies (Legon et al., 2016), it is now possible to explore in great detail the excitatory/inhibitory balance across brain regions in humans, thus helping us to define the conditions for processing SDMs and contributing to our understanding of DA. Another line of research that could be translated to human patients deals with the potential role of neuronal oscillations in DA. To date, low frequency oscillations have been largely ignored, with most work focusing on theta, alpha, beta and gamma oscillations. However, focusing on delta oscillations could be more relevant for understanding SDM and its role in DA. Lastly, although we only touched on the role of microRNAs as regulators of cellular states, these molecules might show specific profiles in individuals with a history of traumatic stress associated with DA.

In our view, important remaining questions, both for animal and human researchers, relate to the mechanisms by which inaccessible stress-related memories might contribute to psychopathology. If consciously accessible (typically cortically processed) memories of trauma can have debilitating consequences for social behavior, affective behavior, and autonomic function, as they do for PTSD patients, there is no reason to believe that inaccessible memories (typically subcortically processed) would not have similar consequences in DA patients. In fact, one might expect inaccessible memories to have even stronger adverse effects, given the neuroanatomical proximity and connectivity to the amygdala, hypothalamus and other centers for socio-affective and autonomic regulation. Another important issue is under what conditions their successful retrieval might be beneficial or detrimental for patients. Lastly, we know little about extinction of affective (e.g., fear) and behavioral (e.g., avoidance) symptoms related to SDM or DA. More research in this area is needed, particularly in view of the fact that extinction processes themselves are sometimes state-dependent and even facilitated under increased stress levels (Self and Choi, 2004). From a basic science perspective, novel circuit approaches in neurobiology should be able to address these questions by determining the relationship between SDM and affective circuits, in the same way as they have already been applied to research with accessible memories (Ramirez et al., 2015). As already indicated, one of the challenges that we face in investigating these issues experimentally relates to the ethical problems associated with applying extreme stress to animals and humans. In animal research, this can, to some extent be circumvented by working with genetically susceptible individuals that are more likely to engage memory-suppressing mechanisms even when stress is not excessive. In the human domain, we suspect the best approach would be to invest more heavily in genetic, epigenetic, imaging and behavioral studies in patients as a way of providing additional support to existing psychotherapies.

\section{REVISITING SKEPTICISM CONCERNING DA}

In this final section, we shall make a few observations relating to skepticism surrounding the notion of DA itself (Pope et al., 1998; Piper and Merskey, 2004; McNally, 2007). In doing so, we will focus on issues pertaining to information processing and memory in general. Objections to the idea of DA can be summarized as follows: (1) encoding inaccessible memories is not a "natural capacity" of the brain; (2) there are no recovered memories because the memories in question were never lost or repressed-they were simply forgotten or not thought about in the ordinary way; (3) recovered memories are false memories; (4) there are no recovered memories because there never were any memories to lose or repress in the first place-they were never formed (e.g., due to infantile amnesia); (5) known biological mechanisms of memory show that stress can only enhance memory; and (6) if traumatic stress triggers $\mathrm{DA}$, why is it found only in some traumatized individuals? We should note that whereas we are not convinced by some of the arguments adduced by DA skeptics, we nevertheless agree that raising such questions is a legitimate enterprise especially given the lack of rigorous analyses of DA in early reports.

With respect to objection (1) that encoding inaccessible memories is not a "natural capacity" of the brain (e.g., McNally, 2007), our response is unquestionably that it is, and our conviction is not based only on brain research. In fact, highly restricted state-dependent access to information is a cellular phenomenon, evolutionarily evident as early as in plants $\mathrm{Ku}$ et al., 2015). Under extreme (abiotic) stress, plants completely shift their genetic expression program in such a way as to preclude access to mechanisms that regulate their normal behavior, instead allowing access to mechanisms that give rise to stress-specific adaptive behavior. Moreover, this massive reversal of information processing is regulated by microRNA molecules (Sunkar et al., 2012), which also play a prominent role in neurons and have been implicated in SDM by virtue of regulating GABA receptor levels (Jovasevic et al., 2015). Importantly, brain microRNAs can reach the blood, and can thus contribute to the assessment of processes taking place in the brain. Although more research is needed in this area, the field has already moved significantly towards understanding the relationship between blood microRNAs and psychopathologies such as schizophrenia and depression (Moreau et al., 2011). Similar studies in patients with DA could be helpful as an auxiliary diagnostic tool.

Of the remaining candidate objections two are particularly worth addressing-the "dissociated memories are merely forgotten memories" claim (2), and (3) the "recovered memories are false memories" claim. Defenders of DA as a bone fide condition claim that DA does not follow the rules of ordinary forgetting. In particular, they argue that DA is 
more likely to occur after repeated episodes, which ordinarily improves remembering, and that unlike normal memory processes, dissociated memories are not sensitive to reminders (Spiegel et al., 2011b). Similarly, Waller et al. (1996) proposed that discontinuity in consciousness associated with DA is an extreme deviation from normality. They proposed that psychopathological dissociation is separate from the normative continuum of dissociation and that rather than a simple cluster of scores at the high end of a continuum, pathological DA is a completely separate construct. This position is taken by others as well (van der Kolk and Fisler, 1995; Harrison et al., 2017), and has received recent support from animal research on SDM. Nevertheless, critics of traumatic amnesia argue that there is nothing special about memories that cannot be accessed during DA (Shobe and Kihlstrom, 1997) and that in fact they represent nothing more than mere forgetting. Unfortunately, the issue of forgetting is difficult to adjudicate because the notions of "normal remembering and forgetting" remain vague. As discussed earlier, the term forgetting is ambiguous and quite generic because it can refer to both degraded and inaccessible memories. From a neurobiological perspective, there have been important advances in defining mechanistically different types of forgetting: neurogenesis-based forgetting, interference-based forgetting, and intrinsic forgetting (Davis and Zhong, 2017). However, all of these kinds of forgetting assume partial or complete memory loss. Regarding blocked access forgetting, other difficulties arise because there can be different causes of the memory deficits, including failure of retrieval mechanisms (Ouyang and Thomas, 2005), reactivation-induced memory modifications (Nader et al., 2000; Alberini et al., 2006), or state-dependence (Gisquet-Verrier et al., 2015; Radulovic et al., 2017). In any case, specifying the nature of normal forgetting seems to be essential for developing further the argument on forgetfulness in amnesia.

The objection (3) that recovered traumatic memories are false memories similarly suffers from vagueness as to what it is for a memory to be a false memory. Is the objection the strong, but implausible, claim that "recovered" memories are all complete fabrications, or is it the weaker claim that some (perhaps even many) of the details of such memories are erroneous? As indicated in the section on memory fidelity, there is nothing abnormal about memories being false, especially in the second sense. The problem of false memories is more serious as a societal and legal problem, but from the standpoint of memory research most of our memories are to a certain degree false. Although fragmented memories might indeed render trauma survivors more prone to form trauma-relevant false memories (Jacobs and Nadel, 1998) it is a bit puzzling why this becomes an issue only for memories that were forgotten and then remembered, as in DA, and not for all of our memories, especially given the ease with which false memories can be produced, even in the laboratory (Roediger and McDermott, 1995; Wade et al., 2007). To complicate matters further, in parallel with false memories, another, apparently very robust and quite opposite phenomenon becomes pronounced as we age. This is the "reminiscence bump" whereby in older people early memories start to be over-represented in what they spontaneously recall, resulting in remembering even those early life events to which access has long been denied (Koppel and Rubin, 2016). Thus, rather than taking sides in this debate, it might be more helpful to intensify research in psychology and neurobiology that attempts to differentiate false from veridical memories. A recent study suggests that this might be possible, by demonstrating discrete patterns of brain activity during processing of true, imagined, and high stressrelated memories (Palmieri et al., 2014).

We believe that the objection (5) that stress only enhances memory can be rejected on the basis of neuroscientific evidence for memory suppressing effects of stress (recently reviewed, Moreira et al., 2016), and the objection (4) that there can be no recovered memories because such putative memories were never formed in the first place, is an untestable proposition and therefore devoid of empirical content. Neither we, nor the proponents of such a claim, can ever provide any evidence of the non-existence of something.

Finally, Piper and Merskey (2004) have expressed a general concern about the relationship between trauma and dissociative disorders because, among other things, (6) many individuals experience trauma but do not develop the disorder. Although a number of retrospective and prospective studies have identified the role of chronic childhood trauma in the development of dissociative disorders, trauma, although necessary, has never been considered to be sufficient for their emergence (Sanders and Giolas, 1991; Ogawa et al., 1997). We do not find this to be a compelling objection partly because it is now well established that there is no unitary response to traumatic stress. But more important, this kind of wholesale rejection of individual differences is inconsistent with the fact that many genetic, epigenetic and environmental factors confer susceptibility, resilience, or resistance to different psychopathologies.

To sum up, we believe that strong clinical evidence, compelling neurobiological evidence, and well-grounded theoretical arguments all lead to the conclusion that DA is a real phenomenon and that modern advances might enable us to distinguish between legitimate cases of DA on the one hand and contrived cases on the other. Furthermore, we believe that we have provided some convincing reasons for supposing that state dependence constitutes a good explanation of at least some of the mechanisms that underlie DA.

\section{AUTHOR CONTRIBUTIONS}

JR wrote parts of the overview, the section on neurobiological mechanism and conclusion. RL provided the case study and associated material, and $\mathrm{AO}$ wrote substantial parts of the overview and conclusion and the section on cognitive perspective. All authors listed have made a substantial, direct and intellectual contribution to the work, and approved it for publication.

\section{FUNDING}

This work was supported by National Institute of Health/National Institute of Mental Health (NIH/NIMH) grants MH078064 and MH108837 to JR. 


\section{ACKNOWLEDGMENTS}

We thank Dr. Michael Kopelman (Kings College, London) for making us aware of the dearth of facilities dedicated to research

\section{REFERENCES}

Ahnaou, A., and Drinkenburg, W. H. (2016). Simultaneous changes in sleep, qEEG, physiology, behaviour and neurochemistry in rats exposed to repeated social defeat stress. Neuropsychobiology 73, 209-223. doi: 10.1159/000446284

Alberini, C. M., Milekic, M. H., and Tronel, S. (2006). Mechanisms of memory stabilization and de-stabilization. Cell. Mol. Life Sci. 63, 999-1008. doi: 10.1007/s00018-006-6025-7

Albrecht, A., Ivens, S., Papageorgiou, I. E., Caliskan, G., Saiepour, N., Bruck, W., et al. (2016). Shifts in excitatory/inhibitory balance by juvenile stress: a role for neuron-astrocyte interaction in the dentate gyrus. Glia 64, 911-922. doi: 10.1002/glia.22970

Anderson, M. C., and Green, C. (2001). Suppressing unwanted memories by executive control. Nature 410, 366-369. doi: 10.1038/35066572

Anderson, M. C., Ochsner, K. N., Kuhl, B., Cooper, J., Robertson, E., Gabrieli, S. W., et al. (2004). Neural systems underlying the suppression of unwanted memories. Science 303, 232-235. doi: 10.1126/science.10 89504

Anderson, R. C., and Ortony, A. (1975). On putting apples into bottles-a problem of polysemy. Cogn. Psychol. 7, 167-180. doi: 10.1016/0010-0285(75)90008-0

Barron, H. C., Vogels, T. P., Behrens, T. E., and Ramaswami, M. (2017). Inhibitory engrams in perception and memory. Proc. Natl. Acad. Sci. U S A 114, 6666-6674. doi: 10.1073/pnas.1701812114

Barron, H. C., Vogels, T. P., Emir, U. E., Makin, T. R., O'Shea, J., Clare, S., et al. (2016). Unmasking latent inhibitory connections in human cortex to reveal dormant cortical memories. Neuron 90, 191-203. doi: 10.1016/j.neuron.2016. 02.031

Bartlett, F. (1932). Remembering: A Study in Experimental and Social Psychology. New York, NY: Cambridge University Press.

Benoit, R. G., Hulbert, J. C., Huddleston, E., and Anderson, M. C. (2015). Adaptive top-down suppression of hippocampal activity and the purging of intrusive memories from consciousness. J. Cogn. Neurosci. 27, 96-111. doi: 10.1162/jocn_a_00696

Berridge, C. W., and Waterhouse, B. D. (2003). The locus coeruleusnoradrenergic system: modulation of behavioral state and state-dependent cognitive processes. Brain Res. Rev. 42, 33-84. doi: 10.1016/s0165-0173(03) 00143-7

Bonanno, G. A., Noll, J. G., Putnam, F. W., O’Neill, M., and Trickett, P. K. (2003). Predicting the willingness to disclose childhood sexual abuse from measures of repressive coping and dissociative tendencies. Child Maltreat. 8, 302-318. doi: $10.1177 / 1077559503257066$

Bransford, J. D., and Franks, J. J. (1971). The abstraction of linguistic ideas. Cogn. Psychol. 2, 331-350. doi: 10.1016/0010-0285(71)90019-3

Breuer, J., and Freud, S. (1955). Studies on Hysteria. London: Hogarth Press.

Brewin, C. R., Dalgleish, T., and Joseph, S. (1996). A dual representation theory of posttraumatic stress disorder. Psychol. Rev. 103, 670-686. doi: 10.1037/0033295x.103.4.670

Briere, J., Scott, C., and Weathers, F. (2005). Peritraumatic and persistent dissociation in the presumed etiology of PTSD. Am. J. Psychiatry 162, 2295-2301. doi: 10.1176/appi.ajp.162.12.2295

Brna, T. G. Jr., and Wilson, C. C. (1990). Psychogenic amnesia. Am. Fam. Physician $41,229-234$

Carr, M. F., and Frank, L. M. (2012). A single microcircuit with multiple functions: state dependent information processing in the hippocampus. Curr. Opin. Neurobiol. 22, 704-708. doi: 10.1016/j.conb.2012.03.007

Cassel, A., and Humphreys, K. (2016). Psychological therapy for psychogenic amnesia: successful treatment in a single case study. Neuropsychol. Rehabil. 26, 374-391. doi: 10.1080/09602011.2015.1033431

Choi, K. R., Seng, J. S., Briggs, E. C., Munro-Kramer, M. L., GrahamBermann, S. A., Lee, R. C., et al. (2017). The dissociative subtype of posttraumatic stress disorder (PTSD) among adolescents: co-occurring PTSD, depersonalization/derealization and other dissociation symptoms. J. Am. and treatment of dissociative amnesia in the USA. The article was stimulated by emails from sufferers struggling to remember important parts from their lives while trying to cope with anxiety, depression and problems in their interpersonal relationships.

Acad. Child Adolesc. Psychiatry 56, 1062-1072. doi: 10.1016/j.jaac.2017. 09.425

Coifman, K. G., Bonanno, G. A., Ray, R. D., and Gross, J. J. (2007). Does repressive coping promote resilience? Affective-autonomic response discrepancy during bereavement. J. Pers. Soc. Psychol. 92, 745-758. doi: 10.1037/0022-3514. 92.4.745

Colman, A. (2009). A Dictionary of Psychology. New York, NY: Oxford University Press.

Colpaert, F. C., Koek, W., and Bruins Slot, L. A. (2001). Evidence that mnesic states govern normal and disordered memory. Behav. Pharmacol. 12, 575-589. doi: 10.1097/00008877-200112000-00002

Crews, F. C. (1995). The Memory Wars: Freud's Legacy in Dispute. New York, NY: New York Review of Books.

Curtois, C. A., and Ford, J. D. (2009). Treating Complex Traumatic Stress Disorders: An Evidence-Based Guide. New York, NY: Guilford Press.

Davis, R. L., and Zhong, Y. (2017). The biology of forgetting-A perspective. Neuron 95, 490-503. doi: 10.1016/j.neuron.2017.05.039

Drouet, J. B., Fauvelle, F., Maunoir-Regimbal, S., Fidier, N., Maury, R., Peinnequin, A., et al. (2015). Differences in prefrontal cortex GABA/glutamate ratio after acute restraint stress in rats are associated with specific behavioral and neurobiological patterns. Neuroscience 285, 155-165. doi: 10.1016/j. neuroscience.2014.10.058

Eich, E. (1995). Mood as a mediator of place dependent memory. J. Exp. Psychol. Gen. 124, 293-308. doi: 10.1037/0096-3445.124.3.293

Eichenbaum, H. (2000). A cortical-hippocampal system for declarative memory. Nat. Rev. Neurosci. 1, 41-50. doi: 10.1038/35036213

Fanselow, M. S. (1990). Factors governing one-trial contextual conditioning. Anim. Learn. Behav. 18, 264-270. doi: 10.3758/bf03205285

Fetkewicz, J., Sharma, V., and Merskey, H. (2000). A note on suicidal deterioration with recovered memory treatment. J. Affect. Disord. 58, 155-159. doi: 10.1016/s0165-0327(98)00193-1

Foa, E. B. (2006). Psychosocial therapy for posttraumatic stress disorder. J. Clin. Psychiatry 67, 40-45. Available online at: http://www.breathofhopefoundation.org/articles/v67s0206_PsychoSocialTx.pdf

Froemke, R. C. (2015). Plasticity of cortical excitatory-inhibitory balance. Annu. Rev. Neurosci. 38, 195-219. doi: 10.1146/annurev-neuro-071714-034002

Gale, G. D., Anagnostaras, S. G., Godsil, B. P., Mitchell, S., Nozawa, T., Sage, J. R., et al. (2004). Role of the basolateral amygdala in the storage of fear memories across the adult lifetime of rats. J. Neurosci. 24, 3810-3815. doi: 10.1523/JNEUROSCI.4100-03.2004

Garner, A. R., Rowland, D. C., Hwang, S. Y., Baumgaertel, K., Roth, B. L., Kentros, C., et al. (2012). Generation of a synthetic memory trace. Science 335, 1513-1516. doi: 10.1126/science.1214985

Girden, E., and Culler, E. (1937). Conditioned responses in curarized striate muscle in dogs. J. Comp. Psychol. 23, 261-274. doi: 10.1037/h0058634

Gisquet-Verrier, P., Lynch, J. F. III., Cutolo, P., Toledano, D., Ulmen, A., Jasnow, A. M., et al. (2015). Integration of new information with active memory accounts for retrograde amnesia: a challenge to the consolidation/reconsolidation hypothesis? J. Neurosci. 35, 11623-11633. doi: 10.1523/JNEUROSCI.1386-15.2015

Godden, D. R., and Baddeley, A. D. (1975). Context-dependent memory in two natural environments: on land underwater. Br. J. Psychol. 66, 325-331. doi: 10.1111/j.2044-8295.1975.tb01468.x

Gradinaru, V., Zhang, F., Ramakrishnan, C., Mattis, J., Prakash, R., Diester, I., et al. (2010). Molecular and cellular approaches for diversifying and extending optogenetics. Cell 141, 154-165. doi: 10.1016/j.cell.2010.02.037

Grillon, C. (2002). Startle reactivity and anxiety disorders: aversive conditioning, context, and neurobiology. Biol. Psychiatry 52, 958-975. doi: 10.1016/s00063223(02)01665-7

Hannula, D. E., and Greene, A. J. (2012). The hippocampus reevaluated in unconscious learning and memory: at a tipping point? Front. Hum. Neurosci. 6:80. doi: 10.3389/fnhum.2012.00080 
Harrison, N. A., Johnston, K., Corno, F., Casey, S. J., Friedner, K., Humphreys, K., et al. (2017). Psychogenic amnesia: syndromes, outcome, and patterns of retrograde amnesia. Brain 140, 2498-2510. doi: 10.1093/brain/awx186

Hasselmo, M. E. (2005). What is the function of hippocampal theta rhythm? - Linking behavioral data to phasic properties of field potential and unit recording data. Hippocampus 15, 936-949. doi: 10.1002/hipo.20116

Haugaard, J. J. (2004). Recognizing and treating uncommon behavioral and emotional disorders in children and adolescents who have been severely maltreated: somatization and other somatoform disorders. Child Maltreat. 9, 169-176. doi: 10.1177/1077559504264318

Henke, K. (2010). A model for memory systems based on processing modes rather than consciousness. Nat. Rev. Neurosci. 11, 523-532. doi: 10.1038/nrn2850

Hennig-Fast, K., Meister, F., Frodl, T., Beraldi, A., Padberg, F., Engel, R. R., et al. (2008). A case of persistent retrograde amnesia following a dissociative fugue: neuropsychological and neurofunctional underpinnings of loss of autobiographical memory and self-awareness. Neuropsychologia 46, 2993-3005. doi: 10.1016/j.neuropsychologia.2008.06.014

Herman, J. (1992). Trauma and Recovery. New York, NY: Basic Books, Perseus Book Group.

Hill, S. Y., Schwin, R., Powell, B., and Goodwin, D. W. (1973). Statedependent effects of marihuana on human memory. Nature 243, 241-242. doi: $10.1038 / 243241 \mathrm{a} 0$

Jacobs, W. J., and Nadel, L. (1998). Neurobiology of reconstructed memory. Psychol. Public Policy Law 4, 1110-1134. doi: 10.1037/1076-8971.4.4.1110

Janet, P. (1889). L'Automtisme Psychologique. Paris: Alcan.

Jiang, J., Wang, G. Y., Luo, W., Xie, H., and Guan, J. S. (2018). Mammillary body regulates state-dependent fear by alternating cortical oscillations. Sci. Rep. 8:13471. doi: 10.1038/s41598-018-31622-z

Jovasevic, V., Corcoran, K. A., Leaderbrand, K., Yamawaki, N., Guedea, A. L., Chen, H. J., et al. (2015). GABAergic mechanisms regulated by miR-33 encode state-dependent fear. Nat. Neurosci. 18, 1265-1271. doi: 10.1038/nn.4084

Kesner, R. P., and Rolls, E. T. (2015). A computational theory of hippocampal function and tests of the theory: new developments. Neurosci. Biobehav. Rev. 48, 92-147. doi: 10.1016/j.neubiorev.2014.11.009

Kikuchi, H., Fujii, T., Abe, N., Suzuki, M., Takagi, M., Mugikura, S., et al. (2010). Memory repression: brain mechanisms underlying dissociative amnesia. J. Cogn. Neurosci. 22, 602-613. doi: 10.1162/jocn.2009.21212

Kim, J. J., and Fanselow, M. S. (1992). Modality-specific retrograde amnesia of fear. Science 256, 675-677. doi: 10.1126/science.1585183

Knyazev, G. G. (2012). EEG delta oscillations as a correlate of basic homeostatic and motivational processes. Neurosci. Biobehav. Rev. 36, 677-695. doi: 10.1016/j.neubiorev.2011.10.002

Kolassa, I. T., Wienbruch, C., Neuner, F., Schauer, M., Ruf, M., Odenwald, M., et al. (2007). Altered oscillatory brain dynamics after repeated traumatic stress. BMC Psychiatry 7:56. doi: 10.1186/1471-244x-7-56

Koppel, J., and Rubin, D. C. (2016). Recent advances in understanding the reminiscence bump: the importance of cues in guiding recall from autobiographical memory. Curr. Dir. Psychol. Sci. 25, 135-149. doi: 10.1177/0963721416631955

Ku, Y. S., Wong, J. W., Mui, Z., Liu, X., Hui, J. H., Chan, T. F., et al. (2015). Small RNAs in plant responses to abiotic stresses: regulatory roles and study methods. Int. J. Mol. Sci. 16, 24532-24554. doi: 10.3390/ijms161024532

Legon, W., Punzell, S., Dowlati, E., Adams, S. E., Stiles, A. B., and Moran, R. J. (2016). Altered prefrontal excitation/inhibition balance and prefrontal output: markers of aging in human memory networks. Cereb Cortex. 26, 4315-4326. doi: 10.1093/cercor/bhv200

Levy, W. B., and Wu, X. (1996). The relationship of local context codes to sequence length memory capacity. Network 7, 371-384. doi: 10.1088/0954-898x_7_ 2_019

Li, X., Ma, R., Pang, L., Lv, W., Xie, Y., Chen, Y., et al. (2017). Delta coherence in resting-state EEG predicts the reduction in cigarette craving after hypnotic aversion suggestions. Sci. Rep. 7:2430. doi: 10.1038/s41598-017-01373-4

Lisman, J. E., and Jensen, O. (2013). The theta- $\gamma$ neural code. Neuron 77, 1002-1016. doi: 10.1016/j.neuron.2013.03.007

Liu, X., Ramirez, S., and Tonegawa, S. (2014). Inception of a false memory by optogenetic manipulation of a hippocampal memory engram. Philos. Trans. $R$. Soc. Lond. B Biol. Sci. 369:20130142. doi: 10.1098/rstb.2013.0142

Loftus, E. F., and Davis, D. (2006). Recovered memories. Annu. Rev. Clin. Psychol. 2, 469-498. doi: 10.1146/annurev.clinpsy.2.022305.095315
Maras, P. M., Molet, J., Chen, Y., Rice, C., Ji, S. G., Solodkin, A., et al. (2014). Preferential loss of dorsal-hippocampus synapses underlies memory impairments provoked by short, multimodal stress. Mol. Psychiatry 19, 811-822. doi: $10.1038 / \mathrm{mp} .2014 .12$

Markowitsch, H. J., and Staniloiu, A. (2016). Functional (dissociative) retrograde amnesia. Handb. Clin. Neurol. 139, 419-445. doi: 10.1016/b978-0-12-801772-2. 00036-9

Marshall, A. C., and Cooper, N. R. (2017). The association between high levels of cumulative life stress and aberrant resting state EEG dynamics in old age. Biol. Psychol. 127, 64-73. doi: 10.1016/j.biopsycho.2017.05.005

McGaugh, J. L., and Roozendaal, B. (2002). Role of adrenal stress hormones in forming lasting memories in the brain. Curr. Opin. Neurobiol. 12, 205-210. doi: 10.1016/s0959-4388(02)00306-9

McKlveen, J. M., Morano, R. L., Fitzgerald, M., Zoubovsky, S., Cassella, S. N., Scheimann, J. R., et al. (2016). Chronic stress increases prefrontal inhibition: a mechanism for stress-induced prefrontal dysfunction. Biol. Psychiatry 80, 754-764. doi: 10.1016/j.biopsych.2016.03.2101

McNally, R. J. (2007). Dispelling confusion about traumatic dissociative amnesia. Mayo Clin. Proc. 82, 1083-1090. doi: 10.4065/82.9.1083

Meyer, M. A. A., Corcoran, K. A., Chen, H. J., Gallego, S., Li, G., Tiruveedhula, V. V., et al. (2017). Neurobiological correlates of statedependent context fear. Learn. Mem. 24, 385-391. doi: 10.1101/lm.045 542.117

Milad, M. R., Rauch, S. L., Pitman, R. K., and Quirk, G. J. (2006). Fear extinction in rats: implications for human brain imaging and anxiety disorders. Biol. Psychol. 73, 61-71. doi: 10.1016/j.biopsycho.2006.01.008

Moreau, M. P., Bruse, S. E., David-Rus, R., Buyske, S., and Brzustowicz, L. M. (2011). Altered microRNA expression profiles in postmortem brain samples from individuals with schizophrenia and bipolar disorder. Biol. Psychiatry 69, 188-193. doi: 10.1016/j.biopsych.2010.09.039

Moreira, P. S., Almeida, P. R., Leite-Almeida, H., Sousa, N., and Costa, P. (2016). Impact of chronic stress protocols in learning and memory in rodents: systematic review and meta-analysis. PLoS One 11:e0163245. doi: 10.1371/journal.pone.0163245

Nadel, L., and Jacobs, W. J. (1998). Traumatic memory is special. Curr. Dir. Psychol. Sci. 7, 154-157. doi: 10.1111/1467-8721.ep10836842

Nader, K., Schafe, G. E., and LeDoux, J. E. (2000). The labile nature of consolidation theory. Nat. Rev. Neurosci. 1, 216-219. doi: 10.1038/350 44580

Netto, C. A., Oliveira, C. B., Gianlupi, A., Quillfeldt, J., and Izquierdo, I. (1987). Pre-test administration of $\beta$-endorphin, or of electroconvulsive shock reverses the memory disruptive effect of posttraining electroconvulsive shock. Peptides 8, 605-608. doi: 10.1016/0196-9781(87)90032-5

Ogawa, J. R., Sroufe, L. A., Weinfield, N. S., Carlson, E. A., and Egeland, B. (1997). Development and the fragmented self: longitudinal study of dissociative symptomatology in a nonclinical sample. Dev. Psychopathol. 9, 855-879. doi: 10.1017/s0954579497001478

Ouyang, M., and Thomas, S. A. (2005). A requirement for memory retrieval during and after long-term extinction learning. Proc. Natl. Acad. Sci. U S A 102, 9347-9352. doi: 10.1073/pnas.0502315102

Overton, D. A. (1991). Historical context of state dependent learning and discriminative drug effects. Behav. Pharmacol. 2, 253-264. doi: 10.1097/00008877-199109000-00002

Palmieri, A., Calvo, V., Kleinbub, J. R., Meconi, F., Marangoni, M., Barilaro, P., et al. (2014). "Reality" of near-death-experience memories: evidence from a psychodynamic and electrophysiological integrated study. Front. Hum. Neurosci. 8:429. doi: 10.3389/fnhum.2014.00429

Pastalkova, E., Itskov, V., Amarasingham, A., and Buzsáki, G. (2008). Internally generated cell assembly sequences in the rat hippocampus. Science 321, 1322-1327. doi: 10.1126/science. 1159775

Phillips, R. G., and LeDoux, J. E. (1992). Differential contribution of amygdala and hippocampus to cued and contextual fear conditioning. Behav. Neurosci. 106, 274-285. doi: 10.1037/0735-7044.106.2.274

Piper, A., and Merskey, H. (2004). The persistence of folly: critical examination of dissociative identity disorder: Part II. The defence and decline of multiple personality or dissociative identity disorder. Addict. Behav. 49, 678-683. doi: 10.1177/070674370404901005

Pope, H. G. Jr., Hudson, J. I., Bodkin, J. A., and Oliva, P. (1998). Questionable validity of 'dissociative amnesia' in trauma victims. Evidence from prospective 
studies. Br. J. Psychiatry 172, 210-215; discussion 216-217. doi: 10.1192/bjp. 172.3.210

Popoli, M., Yan, Z., McEwen, B. S., and Sanacora, G. (2011). The stressed synapse: the impact of stress and glucocorticoids on glutamate transmission. Nat. Rev. Neurosci. 13, 22-37. doi: 10.1038/nrn3138

Putnam, F. (1989). Psychobiology of Dissociation. New York, NY: Springer-Verlag.

Radulovic, J. (2017). Using new approaches in neurobiology to rethink stressinduced amnesia. Curr. Behav. Neurosci. Rep. 4, 49-58. doi: 10.1007/s40473017-0109-6

Radulovic, J., Jovasevic, V., and Meyer, M. A. (2017). Neurobiological mechanisms of state-dependent learning. Curr. Opin. Neurobiol. 45, 92-98. doi: 10.1016/j. conb.2017.05.013

Ramirez, S., Liu, X., Lin, P. A., Suh, J., Pignatelli, M., Redondo, R. L., et al. (2013). Creating a false memory in the hippocampus. Science 341, 387-391. doi: $10.1126 /$ science. 1239073

Ramirez, S., Liu, X., MacDonald, C. J., Moffa, A., Zhou, J., Redondo, R. L., et al. (2015). Activating positive memory engrams suppresses depression-like behaviour. Nature 522, 335-339. doi: 10.1038/nature14514

Rau, V., DeCola, J. P., and Fanselow, M. S. (2005). Stress-induced enhancement of fear learning: an animal model of posttraumatic stress disorder. Neurosci. Biobehav. Rev. 29, 1207-1223. doi: 10.1016/j.neubiorev.2005. 04.010

Rauch, S. L., Shin, L. M., and Phelps, E. A. (2006). Neurocircuitry models of posttraumatic stress disorder and extinction: human neuroimaging research - past, present, and future. Biol. Psychiatry 60, 376-382. doi: 10.1016/j. biopsych.2006.06.004

Rezayof, A., Zarrindast, M. R., Sahraei, H., and Haeri-Rohani, A. (2003). Involvement of dopamine receptors of the dorsal hippocampus on the acquisition and expression of morphine-induced place preference in rats. J. Psychopharmacol. 17, 415-423. doi: 10.1177/02698811031 74005

Roediger, H., and McDermott, K. B. (1995). Creating false memories: remembering words not presented in lists. J. Exp. Psychol. Learn. Mem. Cogn. 21, 803-814. doi: 10.1037/0278-7393.21.4.803

Rudolph, U., and Möhler, H. (2006). GABA-based therapeutic approaches: GABAA receptor subtype functions. Curr. Opin. Pharmacol. 6, 18-23. doi: 10.1016/j.coph.2005.10.003

Ryle, G. (1945). Knowing how and knowing that. Aristotelian Soc. 46, 1-16.

Sanders, B., and Giolas, M. H. (1991). Dissociation and childhood trauma in psychologically disturbed adolescents. Am. J. Psychiatry 148, 50-54. doi: 10.1176/ajp.148.1.50

Sar, V., Alioğlu, F., Akyuz, G., and Karabulut, S. (2014). Dissociative amnesia in dissociative disorders and borderline personality disorder: self-rating assessment in a college population. J. Trauma Dissociation 15, 477-493. doi: 10.1080/15299732.2014.902415

Schimmenti, A., and Caretti, V. (2016). Linking the overwhelming with the unbearable: developmental trauma, dissociation, and the disconnected self. Psychoanal. Psychol. 33, 106-128. doi: 10.1037/a0038019

Self, D. W., and Choi, K. H. (2004). Extinction-induced neuroplasticity attenuates stress-induced cocaine seeking: a state-dependent learning hypothesis. Stress 7 , 145-155. doi: 10.1080/10253890400012677

Sharma, P., Guirguis, M., Nelson, J., and McMahon, T. (2015). A case of dissociative amnesia with dissociative fugue and treatment with psychotherapy. Prim. Care Companion CNS Disord. 17:3. doi: 10.4088/pcc.14l 01763

Shobe, K. K., and Kihlstrom, J. (1997). Is traumatic memory special? Curr. Dir. Psychol. Sci. 6, 70-74. doi: 10.1111/1467-8721.ep11512658

Shohamy, D., and Turk-Browne, N. B. (2013). Mechanisms for widespread hippocampal involvement in cognition. J. Exp. Psychol. Gen. 142, 1159-1170. doi: $10.1037 / \mathrm{a} 0034461$

Sierra, R. O., Cassini, L. F., Santana, F., Crestani, A. P., Duran, J. M., Haubrich, J., et al. (2013). Reconsolidation may incorporate state-dependency into previously consolidated memories. Learn. Mem. 20, 379-387. doi: 10.1101/lm. 030023.112

Spiegel, D., Loewenstein, R. J., Lewis-Fernández, R., Sar, V., Simeon, D., Vermetten, E., et al. (2011a). Dissociative disorders in DSM-5. Depress. Anxiety 28, E17-E45. doi: 10.1002/da.20923
Spiegel, D., Loewenstein, R. J., Lewis-Fernández, R., Sar, V., Simeon, D., Vermetten, E., et al. (2011b). Dissociative disorders in DSM-5. Depress. Anxiety 28, 824-852. doi: 10.1002/da.20874

Squire, L. R. (1992). Memory and the hippocampus: a synthesis from findings with rats, monkeys, and humans. Psychol. Rev. 99, 195-231. doi: 10.1037/0033-295x. 99.2.195

Staniloiu, A., and Markowitsch, H. J. (2012). Towards solving the riddle of forgetting in functional amnesia: recent advances and current opinions. Front. Psychol. 3:403. doi: 10.3389/fpsyg.2012.00403

Staniloiu, A., and Markowitsch, H. J. (2014). Dissociative amnesia. Lancet Psychiatry 1, 226-241. doi: 10.1016/S2215-0366(14)70279-2

Sunkar, R., Li, Y. F., and Jagadeeswaran, G. (2012). Functions of microRNAs in plant stress responses. Trends Plant Sci. 17, 196-203. doi: 10.1016/j.tplants. 2012.01.010

Thomas-Antérion, C. (2017). Dissociative amnesia: disproportionate retrograde amnesia, stressful experiences and neurological circumstances. Rev. Neurol. 173, 516-520. doi: 10.1016/j.neurol.2017.07.007

Thomson, D. M., and Tulving, E. (1970). Associative encoding and retrieval: weak and strong cues. J. Exp. Psychol. 86, 255-262. doi: 10.1037/h0029997

Todorovic, C., Radulovic, J., Jahn, O., Radulovic, M., Sherrin, T., Hippel, C., et al. (2007). Differential activation of CRF receptor subtypes removes stress-induced memory deficit and anxiety. Eur. J. Neurosci. 25, 3385-3397. doi: 10.1111/j.1460-9568.2007.05592.x

Tronson, N. C., Guzman, Y. F., Guedea, A. L., Huh, K. H., Gao, C., Schwarz, M. K., et al. (2010). Metabotropic glutamate receptor 5/homer interactions underlie stress effects on fear. Biol. Psychiatry 68, 1007-1015. doi: 10.1016/j.biopsych. 2010.09.004

Tulving, E. (1972). "Episodic and semantic memory," in Organization of Memory, eds E. Tulving and W. Donaldson (New York, NY: Academic Press), 381-402.

Tulving, E. (1985). Memory and consciousness. Can. Psychol. 26, 1-12. doi: $10.1037 / \mathrm{h} 0080017$

Tulving, E., and Thomson, D. M. (1973). Encoding specificity and retrieval processes in episodic memory. Psychol. Rev. 80, 352-373. doi: $10.1037 / \mathrm{h} 0020071$

van der Kolk, B. A., and Fisler, R. (1995). Dissociation and the fragmentary nature of traumatic memories: overview and exploratory study. J. Trauma. Stress 8 , 505-525. doi: 10.1007/bf02102887

VandenBos, G. (2015). APA Dictionary of Psychology. Washington, DC: American Psychological Association.

Wade, K. A., Sharman, S. J., Garry, M., Memon, A., Mazzoni, G., Merckelbach, H., et al. (2007). False claims about false memory research. Conscious. Cogn. 16, 18-28; discussion 29-30. doi: 10.1016/j.concog.2006.07.001

Waller, N., Putnam, F. W., and Carlson, E. B. (1996). Types of dissociation and dissociative types: a taxometric analysis of dissociative experiences. Psychol. Methods 1, 300-321. doi: 10.1037/1082-989x.1.3.300

Weingartner, H., Adefris, W., Eich, J. E., and Murphy, D. L. (1976). Encodingimagery specificity in alcohol state-dependent learning. J. Exp. Psychol. Hum. Learn 2, 83-87. doi: 10.1037/0278-7393.2.1.83

Whitfield, C. L. (1995). Memory and Abuse: Remembering and Healing the Effects of Trauma. Deerfield Beach, FL: Health Communications.

Wolf, M. R., and Nochajski, T. H. (2013). Child sexual abuse survivors with dissociative amnesia: what's the difference? J. Child Sex Abus. 22, 462-480. doi: $10.1080 / 10538712.2013 .781094$

Zhu, H., and Roth, B. L. (2014). Silencing synapses with DREADDs. Neuron 82, 723-725. doi: 10.1016/j.neuron.2014.05.002

Conflict of Interest Statement: The authors declare that the research was conducted in the absence of any commercial or financial relationships that could be construed as a potential conflict of interest.

Copyright (c) 2018 Radulovic, Lee and Ortony. This is an open-access article distributed under the terms of the Creative Commons Attribution License (CC BY). The use, distribution or reproduction in other forums is permitted, provided the original author(s) and the copyright owner(s) are credited and that the original publication in this journal is cited, in accordance with accepted academic practice. No use, distribution or reproduction is permitted which does not comply with these terms. 